\title{
An overview of the human immune system and the role of interferon regulatory factors (IRFs)
}

\author{
Ashwinder Kaur ${ }^{1}$, Chee-Mun Fang ${ }^{2 *}$ \\ ${ }^{1}$ School of Pharmacy, The University of Nottingham Malaysia, Selangor Darul Ehsan, Malaysia \\ ${ }^{2}$ Division of Biomedical Sciences, School of Pharmacy, The University of Nottingham Malaysia, Selangor Darul Ehsan, \\ Malaysia

\begin{abstract}
The immune system consists of a dynamic network of cells, proteins, tissues, and organs that communicate to provide adequate defense responses against pathogenic agents. The immune system divide into the non-specific (innate) and the specific (adaptive) components, where the interactions between these two arms are intricately regulated. To deploy effective immune responses, immune systems comprise various cells and molecules that communicate with each other via signaling pathways coordinated by gene regulatory networks. The interferon regulatory factors (IRFs) are critical regulators of both the immune system's development and activation of different cells. To better understand the essential components of the normal immune system, this review essentially aims to cover the current knowledge of individual components of the immune system and the important role of IRFs in regulating the immune system.
\end{abstract}

Keywords: Interferon regulatory factor; innate; adpative; transcription factor; $\mathrm{T}$ cells

Received: $9^{\text {th }}$ October 2020

Accepted: $19^{\text {th }}$ October 2020

Published Online: $28^{\text {th }}$ October 2020
* Correspondence: Chee-Mun Fang, Division of Biomedical Sciences, School of Pharmacy, The University of Nottingham Malaysia, Selangor Darul Ehsan, Malaysia; CheeMun.Fang@ nottingham.edu.my

Citation: Kaur A and Fang C-M. An overview of the human immune system and the role of interferon regulatory factors (IRFs). Prog Microbes Mol Biol 2020; 3(1): a0000129. https://doi.org/10.36877/pmmb.a0000129.

\section{INTRODUCTION}

An effective host defense system depends on prompt recognition and response against invaders. Thus, the immune system produces various cells and molecules that communicate with each other via signaling pathways coordinated by gene regulatory networks. In this regard, the immune system, which is the host defense mechanism, is controlled by transcription factors and other elements that activate or repress their target genes in determining cell fate or effector state to ensure effective immune responses ${ }^{[1]}$. This gene regulatory network is controlled by transcription factors such as Interferon Regulatory Factors (IRFs), comprised of 9 family members (IRF1-9) in mammals. Although this family was initially identified in the type I interferon system, subsequent studies have revealed much broader functions performed by IRF members in host defense. The IRFs play crucial roles in the regulation of immune responses ${ }^{[2]}$. IRFs function as central molecules that mediate different signaling pathways to induce the expression of interferons and inflammatory cytokines.

Furthermore, IRFs also regulate immune cells' development and activation and act as a bridge between innate and adaptive responses. The IRFs family possesses a turn-helix turn motif that recognizes DNA consensus, known as the IFN sensitive response element (ISRE), which can be found in the promoters of many genes involved in immune responses ${ }^{[3]}$. The immune responses and pathogenesis of certain diseases correlate with the balance of Th1 and Th2 responses ${ }^{[4,5]}$. For instance, an imbalance of Th1/Th2 responses, with Th1 bias linked to autoimmune diseases such as systemic lupus erythematosus (SLE) and rheumatoid arthritis (RA). On the other hand, Th2-dominated responses are associated with allergic conditions such as asthma. Interestingly, many IRFs are involved in T helper (Th) differentiation. For instance, IRF1, IRF2, and IRF8 are mainly engaged in Th1 differentiation ${ }^{[6,7]}$. Meanwhile, IRF4 that shares several similar biological activities with IRF5 is critical for Th2 cell development ${ }^{[6,7]}$. IRF4 and IRF5, which are both involved in MyD88-dependent Toll-like Receptor (TLR) signaling, found to interact with each other in the induction of proinflammatory cytokines and type I interferons ${ }^{[8]}$. Furthermore, both of these transcription factors are shown to directly regulate Blimp1, a master regulator of plasma differentiation ${ }^{[9,10]}$. Therefore, we aim to provide an overview of the current knowledge of their roles in immune responses and immune cell development.

\section{THE HUMAN IMMUNE SYSTEM}

Immune responses involve recognition of any "nonself" substances, including pathogens. These foreign pathogens or modified particles present in the host body, resulting in activation of cascade complex events 
known as the inflammatory process to eliminate the nonself-substances ${ }^{[11]}$. Following the inflammatory process, the immune system initiates restoration mechanisms involving a series of cellular and molecular events that mitigate the restoration of tissue homeostasis and resolve the inflammatory process ${ }^{[12]}$. On that note, immune responses are tightly regulated, involving various mechanisms to ensure normal homeostasis. When there is an imbalance of the immune system activity, it can lead to diseases such as autoimmune, chronic inflammatory, and cancer that can potentially be life-threatening ${ }^{[13]}$. In general, the immune system can be subdivided into two forms of protection known as innate and adaptive immune responses that work closely together to provide effective host defenses (Figure 1).

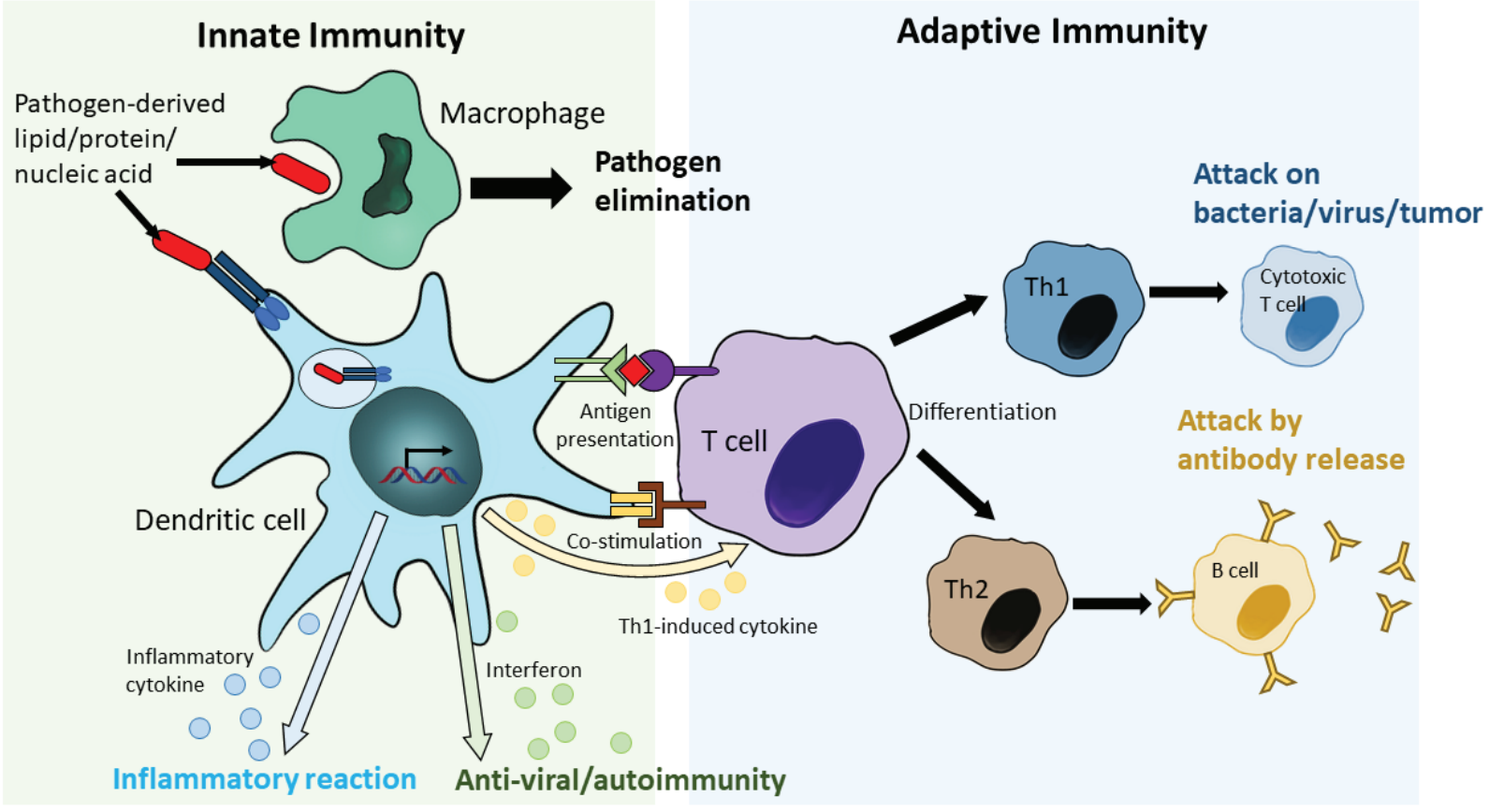

Figure 1. The innate and adaptive immunity in human.

\section{Innate Immunity}

Innate immune response acts as the first line of defense. It is also known as non-specific defense mechanism that responds immediately upon recognizing a diverse array of microbial or "danger" signals due to changes in the homeostasis via the pattern recognition receptors (PRRs) such as Toll-like receptors (TLRs), Nucleotide oligodimerization domain (NOD) -like receptors (NLRs) and Retinoic acid-inducible gene 1 (RIG-1) - like receptors (RLRs), which recognize pathogenassociated molecular patterns (PAMPs) and dangerassociated molecular pattern (DAMPs) ${ }^{[14]}$. These recognition systems elicit distinct cellular responses depending on the nature of stimuli and responding cells. The significant roles of innate responses involve; a) prevent the entry of any foreign substances, b) initiate complement pathway for getting rid of pathogens, c) generation of local inflammatory responses, d) induce phagocytosis and cytotoxicity activities, e) facilitate wound healing and tissue repair and, f) activation of adaptive immune responses through antigen-presenting cells ${ }^{[11,15-17]}$. The innate immune system components consist of physical and chemical barriers, immune cells, and soluble factors that orchestrate rapid immune responses $^{[18]}$. The physical barriers include epithelial layers of skin and the mucosal membrane that protect the external environment and exposure to foreign substances, including pathogens ${ }^{[18]}$. Any breakdown or defect in the physical barrier increases the susceptibility of infection and leads to immune responses. Chemical barriers consist of anti-microbial proteins and peptides, such as defensins permeable to microbes and induce cell death ${ }^{[18]}$. Thus, physical and chemical barriers both play a crucial role in preventing the entry of foreign substances and infection. When pathogens breach protective physical and chemical barriers, they are combated through innate immune cells and soluble proteins. Upon recognition of foreign molecules via the PPRs, innate cells are activated following a cascade of signaling pathways that trigger transcription factors and other proteins to regulate various soluble factors' gene expression to mount defense responses against the pathogens ${ }^{[18,19]}$.

One of the key soluble factors of immune responses is cytokines. Cytokines are peptides that act as mediators in cell communication and signaling. The role of cytokines is not isolated for innate immune responses, as they also play vital roles in adaptive immune responses. Cytokines are broadly classified based on their immune responses and often have overlapping functions ${ }^{[17]}$. Most cytokines are primarily but not exclusively produced by leukocytes called interleukin (IL) ${ }^{[20-22]}$. Interleukins can amplify their production in an autocrine or paracrine manner and induce or inhibit other cytokines' production. They bind to their receptors on the cells that produce them (autocrine) or other cells (paracrine) and modulate the transcriptional program in determining the cells' fate ${ }^{[20]}$. A distinct subset of cytokines named chemotactic cytokines or chemokines is predominantly involved in trafficking activation of 
leukocytes to inflammatory $\operatorname{sites}^{[17,22,23]}$. Concerning immune cells, the cells are developed from hematopoietic stem cells (HSCs) within the bone marrow ${ }^{[24]}$. As these cells mature, they can be differentiated into two main lineages, myeloid progenitor cells (neutrophils, basophils, eosinophils, monocytes, macrophages, mast cells, and dendritic cells) and lymphoid progenitor cells (B cells, T cells, and innate lymphoid cells) ${ }^{[24]}$.

Some of the primary innate immune cells include the granulocytes that contain granules sacs containing enzymes and inflammatory proteins (neutrophils, basophils, eosinophils, and mast cells ${ }^{[24]}$. Among these cells, neutrophils are the most abundant leukocytes whose primary function in host defense is to patrol and guard the immune system against invaders ${ }^{[17,24]}$. While basophils and eosinophils are responsible for defense against helminth and allergic-related diseases through the degranulation process ${ }^{[20,24]}$. Mast cells primarily reside in the tissues rather than in the circulatory, unlike the other granulocytes ${ }^{[20]}$. Mast cells play an important role in triggering inflammatory response as well as participate in wound healing ${ }^{[24]}$.

Meanwhile, another group of innate cells; monocytes, macrophages, and dendritic cells, belong to the mononuclear phagocyte system that plays multiple roles during inflammation ${ }^{[25]}$. Monocytes circulate in the circulatory with a rather short life span and may differentiate into tissue macrophages or dendritic cells depending on the surrounding stimuli ${ }^{[17,25]}$. Both macrophages and dendritic cells are also known as the antigen presenting cells (APCs), as they capable of processing and presenting foreign protein-based molecules (antigens) to lymphocytes ${ }^{[20,26]}$. In terms of protection against virally infected cells and tumor cells, other innate cells known as Natural Killer (NK) cells are well known for its function in killing infected and transformed cells that managed to escape $\mathrm{T}$ cell recognition ${ }^{[24]}$.

\section{Adaptive Immunity}

The adaptive immune response is highly specific. It involves recognizing antigens (foreign agents and particles) via the receptors bound to the surface of $\mathrm{B}$ lymphocytes and $\mathrm{T}$ lymphocytes, which are unique to different antigens ${ }^{[23]}$. This second defense mechanism is a contingent of the innate immune system and initiated in the later onset of infection. It provides excellent defense responses against persistent infection and, importantly, possesses immunological memory ${ }^{[15,23]}$. When the same or closely related antigens are encountered, the immunological memory program is activated, and the adaptive immune system provides rapid and enhanced protection. The adaptive immunity is broadly divided into humoral-mediated immune responses and cell-mediated immune responses coordinated by $\mathrm{B}$ cells and $\mathrm{T}$ cells, respectively ${ }^{[21,23]}$. Humoral mediated immune responses involve the production of antibodies by B cells against soluble antigens such as extracellular microbes and toxins. On the contrary, cell-mediated reactions involve activation of effector $\mathrm{T}$ cells such as cytotoxic $\mathrm{T}$ cells that kill intracellular microbes and tumor cells. These are inaccessible to antibodies and $\mathrm{T}$ helper cells that produce cytokines for modulating other immune cells' function to mount against the antigens.

The principal cells involved in the adaptive immune response are APCs, B, and T-lymphocytes ${ }^{[23]}$. APCs refers to the specialized cells that internalize and process antigen, concomitantly presenting the antigen as peptide within MHC (also known as human leukocyte antigen, HLA, the term designated for humans) on their cell surface ${ }^{[16,23]}$. There are two types of MHC complexes. MHC class I is expressed on all nucleated cells and present peptide antigens derived from intracellular antigens (e.g., viral proteins, autologous proteins, and tumor antigens $)^{[21,23,27]}$. On the contrary, MHC class II expression is predominantly restricted to APCs and presents peptide antigens synthesized from extracellular antigens (e.g., extracellular microbes, toxins, and allergens). Examples of prominent APCs are DCs, macrophages, B cells, and thymic epithelium ${ }^{[27]}$. Antigens processed by dendritic cells are displayed on their MHC class I and class II are capable of activating naïve $\mathrm{T}$ cells into helper $\mathrm{T}$ cells $\left(\mathrm{CD}^{+}\right)$or cytotoxic $\mathrm{T}$ cells $\left(\mathrm{CD} 8^{+}\right)$subsets, respectively ${ }^{[15,26,27]}$. Macrophages and $\mathrm{B}$ cells can also serve as APCs, presenting antigens to T cells during different type of immune responses ${ }^{[26]}$. A subset of DCs known as follicular dendritic cells can present antigens to B cells to establish the humoral immune response ${ }^{[20]}$.

As mentioned previously, B cells and $\mathrm{T}$ cells are the lymphocytes which developed from lymphoid lineage originated from the hematopoietic stem cells (HSCs) that share the same common lymphoid progenitors with innate lymphoid cells (e.g., NK cells). These lymphocytes undergo complex maturation by which they express surface receptors that dictate their functions and phenotypes. Upon recognizing and binding the antigen-specific to their surface receptors, $\mathrm{B}$ and $\mathrm{T}$ cells undergo activation, proliferation (clonal expansion), and differentiation to effector cells and memory cells ${ }^{[11]}$. Unlike B and T cells, innate lymphoid cells are not clonally expressed for specific antigens, serving the innate defense system ${ }^{[23]}$.

B cells comprise several subsets classified based on their ontogeny and anatomical location in $^{[28]}$. For example, B1 and B2 B cells are associated with antibody productions and regulatory $\mathrm{B}$ cells (Bregs), which are essential for suppressing autoimmune and inflammatory responses. B cells' developmental and maturation process involves structural and functional rearrangement of their receptors within the bone marrow ${ }^{[29]}$. B cells express membranebound immunoglobulin (Ig) receptors on their surface and produce soluble antibodies of the receptor's same antigenic specificity. Mature B cells (express membrane-bound IgM and $\mathrm{IgD}$ ) migrate to peripheral lymphoid organs or lymph nodes via the circulatory, where they encounter antigens to establish humoral immunity ${ }^{[28,29]}$. Depending on the nature of antigens encountered and the subset of B cells involved. These B cells can be activated either with the involvement of activated helper $\mathrm{T}$ cells that express CD40L and the cytokines produced by them (T-dependent $\mathrm{B}$ cell activation) or without the involvement of helper T cells (T-independent $\mathrm{B}$ cell activation), which is usually facilitated by TLR stimulation $^{[29,30]}$.

Following the activation of $\mathrm{B}$ cells, the $\mathrm{B}$ cells undergo 
clonal expansion and differentiate into plasma cells that produce IgM and IgD type antibodies designed to mount against the particular antigens ${ }^{[28]}$. Besides, most of the $\mathrm{B}$ cells become effector cells, plasma cells that further interact with other stimuli such as cytokines in the local microenvironment. While the plasma cells capable of producing different classes of antibodies other than IgM and $\operatorname{IgD}(\operatorname{Ig} \mathrm{A}, \operatorname{IgG}$, and $\operatorname{IgE})$ through the process called immunoglobulin class switching ${ }^{[28,29]}$. Some of the B cells become memory cells that preserve the "information" for those successful antibodies generated against the antigen and provide robust protection if the same antigens are encountered ${ }^{[29]}$. The functions of antibodies are to neutralize virulence factors of antigens and enhance the complement pathway and phagocytes' activation to eliminate the antigens ${ }^{[11,18,23]}$. However, antibodies can also contribute to autoimmune diseases' pathogenesis due to disrupted self-tolerance mechanisms leading to the generation of autoantibodies as a consequence of $\mathrm{B}$ cells reacting against self-antigens (particles of host body) ${ }^{[28]}$. Unlike B cells that can recognize antigens in the extracellular spaces, $\mathrm{T}$ lymphocytes have restricted specificity for antigens as they identify and respond to surface-bound antigens displayed on the MHC of APCs.

\section{INTERFERON REGULATORY FACTORS (IRFs) FAMILY}

Transcription factors are key players during gene expression as they facilitate when and what gene is "turn on or off' by binding to DNA sequences, acting as coactivator or co-repressor of the gene response to various signals ${ }^{[31]}$. Structurally they comprise of two domains: a) DNA binding domain that recognizes and bind to DNA sequences, b) activation (effector) domain which interacts with cofactors or other transcription factors ${ }^{[32-34]}$. Some of them also have an additional domain (signalsensing domain) that binds to ligands to modulate their activity response to environmental cues ${ }^{[32,33]}$.

Transcription factors are categorized into several groups based on their DNA binding domain structures and their interaction with DNA sequences ${ }^{[33-35]}$. Some of these transcription factors are known as general transcription factors (GTFs), which are ubiquitous and essential for initiating transcription in the protein-coding gene ${ }^{[31,34]}$. Other transcription factors are either constitutive or inducible and are specific to certain cell types and stages of organism development ${ }^{[31,34]}$. Several of these transcription factors function as master transcriptional regulators in controlling signal responses and specifying cells' lineage ${ }^{[31,36]}$. Also, transcription factors play a crucial role in interacting with histone proteins, which influence chromatin state and establish the environment that allows interactions for activation or repression of gene transcription ${ }^{[33,37]}$. One group of transcription factors that have been extensively studied for their crucial role in regulating gene networks in the immune system is the Interferon Regulatory Factors (IRFs), which possess the turn-helix turn motif ${ }^{[38]}$.

The first IRFs member, IRF1, was initially identified as a factor that binds to the human interferon's upstream regulatory region (IFN) $\beta$ gene and induced its expression ${ }^{[39]}$. Over decades, the family of IRFs has been expanded to ten members, to which IRF1 to 9 are found in mice and humans, whereas IRF10 is only found in chickens ${ }^{[2]}$. Also, several viral IRFs that interact with cellular IRFs have been identified ${ }^{[40]}$. All IRFs consist of two domains, the N-terminal (DNA binding domain) and C-terminal (regulatory domain). The N-terminal binding domain is enriched with five tryptophan repeats that are well conserved among all IRF. This region recognizes a DNA consensus, known as IFN sensitive response element (ISRE) found in the promoters of type 1 IFN, IFNinducible genes, and many other genes involved in immune responses $^{[3]}$. On the other hand, the C-terminal consists of the IRF association domain (IAD) responsible for homoand heteromeric interaction with other family members or transcription factors ${ }^{[38,41]}$. IRFs functions are not limited to, regulating innate and adaptive immune responses. These transcription factors also play a crucial role in controlling type 1 IFN induced by viruses and pathogens involved in the cell cycle, apoptosis, and oncogenesis ${ }^{[2,3,38,41]}$. Some of these IRFs regulate immune cell development and functions ${ }^{[2,6,7,42]}$.

Accumulating data from several studies has shown that IRF5 is a critical mediator in developing Th1 responses associated with the pathogenesis of various diseases, including autoimmune, metabolic, and infectious diseases $^{[43]}$. Conversely, studies have also demonstrated that IRF5 plays a role in inducing Th2 responses ${ }^{[44,45]}$. Strikingly, most studies have attributed IRF5 in regulating Th1/Th2 reactions by altering antigen-presenting cells (e.g., macrophages and dendritic cells) rather than IRF5 intrinsic properties in T cells. Perhaps because early studies reported that expression of IRF5 is barely detected in T cell $^{[46]}$. Nonetheless, recent studies have detected elevated IRF5 expression in parasitic and viral infected T cells ${ }^{[47,48]}$ and have highlighted its direct role in a $\mathrm{T}$ cell subset during chronic parasitic infection ${ }^{[49]}$. Fabié et al. (2018) [49] demonstrated that TLR-7 mediated activation of IRF5 promoted IFN $\gamma^{+}$CD4 $\mathrm{T}$ cell death because of its ability to enhance death receptor 5 (DR5)-induction cell apoptosis in CD4 T, thus promoting persistent $L$. donovani infection. However, the role of $\mathrm{T}$ cell-intrinsic dependent on IRF5 in modulating Th cytokine production remains uncertain.

\section{Roles of IRFs in $\mathrm{T}$ helper differentiation}

Over the years, many IRFs are involved in $\mathrm{T}$ helper differentiation either by modulating the functions of antigen presenting cells or directly altering the transcription of cytokine genes of Th cells ${ }^{[7]}$. IRF1 is a crucial factor in promoting Th1 differentiation and its absence results in predominant Th2 responses as best characterized using experimental mice disease model. For example, IRF1 deficient mice $\left(\mathrm{Irfl}^{-/}\right)$were vulnerable to Leishmania major (L. major) and Listeria monocytogenes (L. monocytogenes) because the host could not control the parasitic infection due to a lack of Th1 responses, which is needed for protection against the intracellular parasitic infections ${ }^{[50,51]}$. Collectively, IRF1, IRF2, and IRF8 directly participated in Th1 differentiation predominantly by regulating the production of IL-12, the Th1 signature cytokine. 
On the other hand, IRF4 is critical for Th2 cell development. Mice lacking IRF4 showed to be able to mount Th1 immune response against $L$. major, but the Th2 development was impaired ${ }^{[59]}$. Also, $\mathrm{CD}^{+} \mathrm{T}$ cells of Irf $4^{-/}$mice were unable to differentiate into Th2 cells and displayed impaired production of Th2 cytokines; IL-4, IL-5, and IL-13 ${ }^{[60]}$. Moreover, overexpression of IRF4 in human Jurkat T cells activated Th2 cytokines' expression in response to mitogenic stimulation ${ }^{[61]}$. Consistently, in the absence of IRF4, IL-4 failed to induce Th2 cytokine, instead induced Th1 response by upregulating IFN $\gamma$ and TNF expression, thereby implying the importance of IRF4 in Th2 differentiation ${ }^{[62]}$. In accordance with the role of IRF4 in Th2 differentiation, Honma et al. $(2008)^{[63]}$ reported that IRF4 plays a dual role in different stages of $\mathrm{CD}^{+} \mathrm{T}$ cells. IRF4 inhibited Th2 cytokine production in naïve $\mathrm{CD}^{+} \mathrm{T}$ cells but induced Th2 cytokine production in effector/ memory $\mathrm{CD}^{+}$cells. However, IRF4 function is restricted to Th2 cell differentiation since several studies have shown that IRF4 is vital in generating Th9, Th17 and Tregs cells ${ }^{[6]}$. Taken together, IRF4 is able to differentially control the Th subsets and act as Th modulator. On the other hand, IRF5 plays a pivotal role in modulating $T$ helper responses by altering the functions of antigen-presenting cells such as macrophages and dendritic cells and influencing the cytokine production drives the Th differentiation as well as participates in activation downstream of TLR-mediated signaling ${ }^{[3,64]}$.

\section{CONCLUSION}

The immune system protects against invaders such as microbial pathogens, toxic agents, and transformed malignant cells. The crosstalk between innate and adaptive immunity ensures an effective host defense system, mediated by various cells and molecules that work dynamically. In terms of $\mathrm{T}$ cells, upon activation, naïve CD4+ T cells acquire the ability to differentiate into several subsets, including $\mathrm{T}$ helper 1 and T helper 2 cells mediated by specific cytokine signaling and transcription factor. The positive vital regulators involve in Th1 differentiation are T-bet, IFN $\gamma /$ STAT1, and IL-12/STAT4; meanwhile, for Th2 differentiation are GATA3 and IL-4/STAT6. Besides the master transcription factor, several other transcription factors such as the RUNX3, Hlx, IRF1, Dec2, IRF4, NFAT, Gif-1, c-Maf, and Jun-B are known to cooperate with the subset specific master transcription factor that collectively involved in establishing Th1/Th2 differentiation. Although specific cytokines expressions and transcription factors of $\mathrm{T}$ helper subsets are exclusive to each subset, they can still present in both states and fine-tune the $\mathrm{T}$ helper cells capabilities for polarization when the situation requires. This can be exemplified clearly by IL-2, which is essential for both Th1 and Th2 differentiation. Collectively, the immune cells orchestrate a complex network made of transcription factors, in cooperation with other proteins involved in cytokine signaling. In which subsequently influences the chromatin state of the targeted genes, resulting in activation or repression of the genes, thereby eliciting the required immune responses in a given state.

\section{Authors Contributions}

$\mathrm{AK}$ and $\mathrm{C}-\mathrm{MF}$ performed the literature review and manuscript writing. C-MF conceptualizes the research project.

\section{Conflict of Interest}

The authors declare that there is no conflict of interest in this work.

\section{Acknowledgement}

This project is funded by the Ministry of Higher Education, Malaysia, Fundamental Research Grant Scheme (FRGS/1/2013/SKK01/MUSM/03/03).

\section{References}

1. Singh H, Khan AA, Dinner AR, et al., Gene regulatory networks in the immune system. Trends Immunol, 2014; 35: 211-218.

2. Tamura T, Yanai H, Savitsky D, et al., The IRF family transcription factors in immunity and oncogenesis. Annu Rev Immunol, 2008; 26: $535-584$

3. Honda $\mathrm{K}$ and Taniguchi T, IRFs: Master regulators of signalling by Toll-like receptors and cytosolic pattern-recognition receptors. Nat Rev Immunol, 2006; 6: 644-658.

4. Martino M, Rocchi G, Escelsior A, et al., Immunomodulation mechanism of antidepressants: Interactions between Serotonin/Norepinephrine balance and Th1/Th2 Balance. Curr Neuropharmacol, 2012; 10: 97-123. Kidd P, Th1/Th2 balance: The hypothesis, its limitations, and implications for health and disease. Altern Med Rev, 2003; 8: 223-246.

6. Zhao G-N, Jiang D-S, and $\mathrm{Li} \mathrm{H}$, Interferon regulatory factors: at the crossroads of immunity, metabolism, and disease. Biochim Biophys Acta Mol Basis Dis, 2015; 1852: 365-378.

7. Lohoff $\mathrm{M}$ and Mak TW, Roles of interferon-regulatory factors in T-helper-cell differentiation. Nat Rev Immunol, 2005; 5: 125-135.

8. Negishi H, Ohba Y, Yanai H, et al., Negative regulation of Toll-likereceptor signaling by IRF-4. Proc Natl Acad Sci U S A, 2005; 102: 15989-15994

9. Cretney E, Xin A, Shi W, et al., The transcription factors Blimp-1 and IRF4 jointly control the differentiation and function of effector regulatory T cells. Nat Immunol, 2011; 12: 304-311.

10. Lien C, Fang C-M, Huso D, et al., Critical role of IRF-5 in regulation of B-cell differentiation. Proc Natl Acad Sci U S A, 2010; 107: 4664-4668.

11. Kellie $\mathrm{S}$ and Al-Mansour Z, Overview of the immune system. In: Micro and Nanotechnology in Vaccine Development. William Andrew Publishing; 2017. p 63-81.

12. Chen L, Deng H, Cui H, et al., Inflammatory responses and inflammationassociated diseases in organs. Oncotarget, 2018; 9: 7204-7218.

13. Bagatini MD, Cardoso AM, Reschke CR, et al., Immune system and chronic diseases 2018. J Immunol Res, 2018; 2018: 8653572.

14. Suresh R, Mosser DM, Ablasser A, et al., Pattern recognition receptor in innate immunity, host defense, and immunopathology. Adv Physio Educ, 2013; 37: 284-291.

15. Introduction to the Immune Response. In: Primer to the Immune Response. Mak TW, Saunders ME and Jett BD, editors. 2014, USA: Academic Cell. 3-20.

16. Patel P, Innate and Adaptive Immunity: Barriers and Receptor-Based Recognition. Immunity and Inflammation in Health and Disease. 2018 Academic Press. 3-13.

17. Carrillo JLM, García FPC, Coronado OG, et al., Physiology and pathology of innate immune response against pathogens. In: Physiology and Pathology of Immunology. Rezaei N, ed. 2017, Rijeka: IntechOpen.

18. McDonald DR and Levy O, Innate Immunity. Clin Immunol 2019: 3953.

19. Smale ST, Transcriptional regulation in the innate immune system. Curr Opin Immunol 2012; 24: 51-57.

20. Components of the immune system. In: Primer to the Immune Response. 2014, USA: Academic Cell. 21-54.

21. Carlson BM and Carlson BM, The lymphoid system and immunity. In The Human Body. 2019, USA: Academic Press. 209-239.

22. Noakes PS and Michaelis LJ, Innate and adaptive immunity. In Diet, Immunity and Inflammation. 2013, UK: Woodhead Publishing. 3-33.

23. Chaplin DD, The Human Immune Response. Clin Immunol 2019: 3-17.

24. Lewis DE and Blutt SE, Organization of the Immune System. Clin Immunol 2019: 19-38. 
25. Alikhan MA and Ricardo SD, Mononuclear phagocyte system in kidney disease and repair. Nephrology (Carlton, Vic.) 2013; 18: 81-91.

26. Antigen Processing and Presentation. In: Primer to the Immune Response. 2014, USA: Academic Cell. 161-179.

27. Carty SA, Riese MJ, and Koretzky GA, T-Cell Immunity. Hematol, 2018: 221-239

28. Hoffman W, Lakkis FG, and Chalasani G, B cells, antibodies, and more. Clin J Am Soc Nephro, 2016; 11: 137- 54

29. $\mathrm{Yu} \mathrm{Y-H}$ and Lin K-I, Factors that regulate the generation of antibody-secreting plasma cells. Adv Immunol, 2016; 131: 61-99.

30. Goodnow CC, Vinuesa CG, Randall KL, et al., Control systems and decision making for antibody production. Nat Immunol, 2010; 11: 681-688.

31. Pope SD and Medzhitov R, Emerging principles of gene expression programs and their regulation. Mol Cell, 2018; 71: 389-397.

32. Wu J and Brown M, Epigenetics and epigenomics. Hematol, 2018: $17-24$.

33. Lambert SA, Jolma A, Campitelli LF, et al., The human transcription factors. Cell, 2018; 172: 650-665.

34. Caramori G, Nucera F, Coppolino I, et al., Transcription factors. In :Reference Module in Biomedical Sciences. 2020, USA: Elsevier.

35. Wingender $\mathrm{E}$, Schoeps $\mathrm{T}$, Haubrock $\mathrm{M}$, et al., TFClass: A classification of human transcription factors and their rodent orthologs. Nucleic Acids Res, 2015; 43: D97-D102.

36. Lee TI and Young RA, Transcriptional regulation and its misregulation in disease. Cell, 2013; 152: 1237-1251.

37. Gibcus JH and Dekker J, The context of gene expression regulation. F1000 Biol Rep, 2012; 4

38. Taniguchi T, Ogasawara K, Takaoka A, et al., IRF family of transcription factors as regulators of host defense. Annu Rev Immunol, 2001; 19: 623-655.

39. Miyamoto M, Fujita T, Kimura Y, et al., Regulated expression of a gene encoding a nuclear factor, IRF-1, that specifically binds to IFN-beta gene regulatory elements. Cell, 1988; 54: 903-913.

40. Lee H-R, Kim MH, Lee J-S, et al., Viral interferon regulatory factors. J Interferon Cyotkine Res, 2009; 29: 621-627.

41. Honda K, Takaoka A, and Taniguchi T, Type I interferon [corrected] gene induction by the interferon regulatory factor family of transcription factors. Immunity, 2006; 25 : 349-360.

42. Battistini A, Interferon regulatory factors in hematopoietic cell differentiation and immune regulation. J Interferon Cytokine Res, 2009; 29: 765-780.

43. Kaur A, Lee L-H, Chow S-C, et al., IRF5-mediated immune responses and its implications in immunological disorders. Int Rev Immunol, 2018; 37(5): 229-248.

44. Saigusa R, Asano Y, Taniguchi T, et al., Multifaceted contribution of the TLR4-activated IRF5 transcription factor in systemic sclerosis. Proc Natl Acad Sci U S A, 2015; 112: 15136-15141.

45. $\mathrm{Xu} \mathrm{Y}$, Lee PY, Li Y, et al., Pleiotropic IFN-dependent and -independent effects of irf5 on the pathogenesis of experimental lupus. J Immunol, 2012; 188: 4113-4121.

46. Mancl ME, Hu G, Sangster-Guity N, et al., Two discrete promoters regulate the alternatively spliced human interferon regulatory factor-5 isoforms. Multiple isoforms with distinct cell type-specific expression, localization, regulation, and function. J Biol Chem, 2005; 280: 21078-21090.
47. Ishikawa $\mathrm{C}$, Senba M, Barnes BJ, et al., Constitutive expression of IRF-5 in HTLV-1-infected T cells. Int J Oncol, 2015; 47: 361-369.

48. Paun A, Bankoti R, Joshi T, et al., Critical role of IRF-5 in the development of T helper 1 responses to Leishmania donovani infection. PLoS Pathog, 2011; 7: e1001246.

49. Fabié A, Mai LT, Dagenais-Lussier X, et al., IRF-5 promotes cell death in CD4 T cells during chronic infection. Cell Rep, 2018; 24: $1163-$ 1175 .

50. Lohoff M, Ferrick D, Mittrucker HW, et al., Interferon regulatory factor-1 is required for a $\mathrm{T}$ helper 1 immune response in vivo. Immunity, 1997; 6: 681-689.

51. Taki S, Sato T, Ogasawara K, et al., Multistage regulation of Th1-type immune responses by the transcription factor IRF-1. Immunity, 1997; 6: 673-679.

52. Seidl A, Panzer M, and Voehringer D, Protective immunity against the gastrointestinal nematode Nippostrongylus brasiliensis requires a broad T-cell receptor repertoire. Immunol, 2011; 134(2): 214-223.

53. Coccia EM, Passini N, Battistini A, et al., Interleukin-12 induces expression of interferon regulatory factor- 1 via signal transducer and activator of transcription-4 in human T helper type 1 cells. J Biol Chem, 1999; 274: 6698-6703.

54. Galon J, Sudarshan C, Ito S, et al., IL-12 induces IFN regulating factor-1 (IRF-1) gene expression in human NK and T cells. J Immunol, 1999; 162: 7256-7262.

55. Kano S-i, Sato K, Morishita Y, et al., The contribution of transcription factor IRF1 to the interferon-gamma-interleukin 12 signaling axis and TH1 versus TH-17 differentiation of CD4+ T cells. Nat Immunol, 2008; 9: 34-41.

56. Lohoff M, Duncan GS, Ferrick D, et al., Deficiency in the transcription factor interferon regulatory factor (IRF)-2 leads to severely compromised development of natural killer and Thelper type 1 cells. J Exp Med, 2000; 192: 325-336.

57. Elser B, Lohoff M, Kock S, et al., IFN-gamma represses IL-4 expression via IRF-1 and IRF-2. Immunity, 2002; 17: 703-712.

58. Scharton-Kersten T, Contursi C, Masumi A, et al., Interferon consensus sequence binding protein-deficient mice display impaired resistance to intracellular infection due to a primary defect in Interleukin $12 \mathrm{p} 40$ induction. J Exp Med, 1997; 186: 1523-1534.

59. Tominaga N, Ohkusu-Tsukada K, Udono H, et al., Development of Th1 and not Th2 immune responses in mice lacking IFN-regulatory factor-4. Int Immunol, 2003; 15: 1-10.

60. Rengarajan J, Interferon regulatory factor 4 (IRF4) interacts with NFATc2 to modulate Interleukin 4 gene expression. J Exp Med, 2002; 195: 1003-1012.

61. Hu C-M, Jang SY, Fanzo JC, et al., Modulation of T cell cytokine production by interferon regulatory factor-4. J Biol Chem, 2002; 277: 49238-49246.

62. Lohoff M, Mittrücker H-W, Prechtl S, et al., Dysregulated Thelper cell differentiation in the absence of interferon regulatory factor 4 . Proc Natl Acad Sci U S A, 2002; 99: 11808-11812.

63. Honma K, Kimura D, Tominaga N, et al., Interferon regulatory factor 4 differentially regulates the production of $\mathrm{Th} 2$ cytokines in naive vs. effector/memory CD4+ T cells. Proc Natl Acad Sci U S A, 2008; 105: 15890-15895.

64. Ouyang X, Negishi H, Takeda R, et al., Cooperation between MyD88 and TRIF pathways in TLR synergy via IRF5 activation. Biochem Biophys Res Commun, 2007; 354: 1045-1051. 